\title{
IgG4-related Disease - A Patient with Multiple Organ Involvement
}

\section{Miroslav Průcha, Lenka Sedláčková}

Department of Clinical Biochemistry, Haematology and Immunology, $\mathrm{Na}$ Homolce Hospital, Prague, Czech Republic

Received March 27, 2017; Accepted August 28, 2017.

Key words: IgG4 - Fibrosis - Sclerosis - Immunosuppressive therapy

Abstract: IgG4-related diseases represent a heterogeneous group of conditions characterised by elevated serum IgG4 levels and fibrotic or sclerosing changes in the affected organs or systems accompanied by lgG4-positive plasma cells. A disease associated with lgG4 may affect virtually any organ - salivary glands, periorbital tissue, kidneys, lungs, meninges, aorta, prostate, pericardium or skin. Histopathological findings are uniform, characterised by a major lymphoplasmocytic infiltrate and the presence of lgG4-producing plasma cells, irrespective of the affected site. It can be difficult to establish a correct diagnosis due to the lack of clinical symptoms. Treatment with immunosuppressive drugs provides good results and requires interdisciplinary cooperation.

Mailing Address: Assoc. Prof. Miroslav Průcha, MD., PhD., Department of Clinical Biochemistry, Haematology and Immunology, Na Homolce Hospital, Roentgenova 2, 15030 Prague 5, Czech Republic; Phone: +420 257273 051; e-mail: miroslav.prucha@homolka.cz 


\section{Introduction}

IgG4-related disease represents a relatively newly defined condition comprised of a collection of disorders characterised by IgG4 hypergammaglobulinaemia and the presence of IgG4-positive plasma cells in affected organs with fibrotic or sclerotising changes (Kamisawa et al., 2015). IgG4-related disease may affect virtually any organ - salivary glands, periorbital tissue, kidneys, lungs, meninges, aorta, prostate, pericardium, and skin. However, its histopathology is uniform, showing a large lymphoplasmacytic infiltrate enriched in lgG4-positive plasma cells, irrespective of the localisation of involved organs (Mahajan et al., 2014). Mikulicz syndrome is a disease that was originally considered to be a variant of Sjögren's syndrome; at present, it is one of the examples of IgG4-related disease presentation. Inflammatory orbital pseudotumour, Küttner's tumour and idiopathic retroperitoneal fibrosis (Ormond's disease) are other disorders belonging to the group (Brito-Zerón et al., 2014). Being relatively rare, these disorders still have to be taken into consideration when examining patients.

\section{Case report}

A 74-year-old male patient was referred to our immunology outpatient department in order to exclude a systemic inflammatory disease. He had a history of the following diseases: epidemic typhus in 1945, infectious hepatitis A in 1953, myocardial infarction in 2003, and crural vein thrombosis on the left-hand side in 2010. A year and a half ago, the patient experienced orbital oedema and a burning sensation for the first time. Due to professional exposure - the patient grew African violets - allergy was considered; therefore, allergy assessment was performed which revealed nasal polyposis, with no findings of allergy to current inhalation allergens. Orbitopathy with bilateral exophthalmos followed shortly, with no findings supporting an endocrine aetiology. This was followed by the enlargement of salivary and lacrimal glands, with no pronounced xerophthalmia or xerostomia. This was followed by an asymmetrical submandibular gland swelling on the left. The patient had no fever; he reported no subjective complaints: absence of appetite, tiredness or pain upon admission. Objective assessment revealed a clear bilateral eye protrusion with lower eyelid oedema and an asymmetrical submandibular salivary gland swelling on the left. From a cardiopulmonary point of view, the patient was compensated, without any pathology found on the heart, lungs or abdomen. Laboratory findings: ESR $85 / 120$, CRP $<5 \mathrm{mg} / \mathrm{l}$, and biochemical screening showed no significant pathology. Immunology revealed polyclonal hypergammaglobulinaemia, with increased levels of all IgG1-4 subgroups (lgG1 $18.1 \mathrm{~g} /$ /; $\operatorname{lgG} 212.5 \mathrm{~g} /$; $\operatorname{lgG} 35.83 \mathrm{~g} / \mathrm{l} ; \operatorname{lgG} 4$ $5.24 \mathrm{~g} / \mathrm{l}$ ), with no $\mathrm{M}$ gradient, with positive antinuclear antibodies (ANA) with a cytoplasm-type of immunofluorescence at a 1:40 titre, antibodies to extractable nuclear antigens (ENA), anti-neutrophil cytoplasmic antibodies (ANCA), and antiphospholipid antibodies (ACLA, anti-beta GPIb) were negative. Head and neck 
ultrasonography showed a symmetrical salivary gland swelling with reactively enlarged neck lymphatic glands. Head and neck MRI (magnetic resonance imaging) assessment revealed a bilateral symmetric retrobulbar mass outside of the extraocular muscles and parotic and submandibular gland swelling. PET (positron emission tomography) imaging showed locations with glucose hypermetabolism in parotic glands and the left submandibular gland, and in neck and mediastinal lymph nodes. A biopsy examination of the salivary gland was performed which showed significant reactive hyperplasia of the lymphadenoid tissue with lymphatic follicles corresponding to the diagnosis of Mikulicz syndrome. A CT (computed tomography) examination of the abdomen and lower pelvis revealed a nonfunctional left kidney which was slightly wrinkled and had a thick hollow system wall, without dilation, with several cortical cysts. The right kidney had a regular size and structures with normal excretion functions. An increased density was seen around large abdominal vessels, more to the left, which presented itself as infiltration, rather than fibrosis. The patient was diagnosed with an IgG4-associated disease - accompanied by inflammatory orbital pseudotumour, Küttner's tumour, Mikulicz syndrome and Ormond's disease - idiopathic retroperitoneal fibrosis. Corticosteroid therapy was indicated. Hypergammaglobulinaemia and ESR reached normal values in 4 weeks, and orbitopathy disappeared completely. Corticosteroids were administered for seven months and gradually discontinued. The patient had absolutely no subjective complaints and felt excellent. Follow-up examinations were performed every six months, with blood sample collection for immunological examination. Polyclonal hypergammaglobulinaemia (lgG $21 \mathrm{~g} / \mathrm{l})$ and increased lgG4 levels $(4.6 \mathrm{~g} / \mathrm{l})$ were detected in the laboratory results two years later. Again, immunosuppressive therapy using methylprednisolone (Medrol) was administered to the patient - the initial dose of $32 \mathrm{mg}$ was reduced to $2 \mathrm{mg}$ once in two days six months later - which has kept the disease in remission.

\section{Discussion}

IgG4-associated diseases are a newly defined group of disorders, some of which were already known in the past (Table 1). They are characterised by elevated lgG4 serum levels and the presence of plasma cells in the inflammatory infiltrate producing these antibodies. There is a vast variety of clinical presentations and a range of involved organs and systems (Stone et al., 2015). The pathogenic role of lgG4 antibodies is poorly understood (Ebbo et al., 2012). We have relatively vast experience in the diagnosis and treatment of Ormond's disease - idiopathic retroperitoneal fibrosis - the treatment of which is provided in cooperation with an urologist and the vascular surgery department (Prúcha et al., 2016). Our case report shows vast multiple organ involvement of various organs and systems. Mikulicz syndrome and inflammatory orbital pseudotumour were presented as clinically dominant entities. Idiopathic retroperitoneal fibrosis that was discovered 


\section{Table 1 - IgG4-associated diseases}

- Mikulicz syndrome with the involvement of salivary and lacrimal glands

- Küttner's tumour affecting submandibular glands

- Riedel's thyroiditis

- Inflammatory pseudotumours affecting orbits, lungs, kidney

- Mediastinal fibrosis

- Multifocal fibrosclerosis affecting aorta, thyroid gland, retroperitoneal space

- Ormond's diseases - idiopathic retroperitoneal fibrosis

- Periarteritis and periaortitis

- Inflammatory aortic aneurysm

- Idiopathic hypocomplementemic tubulointerstitial nephritis

in a relatively inactive stage led to the loss of one kidney without the patient noticing. From the practical point of view, it is necessary to stress that patients with Ormond's disease present themselves with largely non-specific complaints tiredness, sometimes subfebrile temperature, abdominal pain, and back pain. A common basic biochemical examination does not usually reveal any pathology, except for increased ESR and elevated C-reactive protein levels in half of the cases. In the absence of clear clinical symptomatology, diagnosis can be made based on ultrasound abdominal examination which can reveal the involvement of the kidney hollow system or abdominal aorta during an active disease. The gold standard of diagnosis is histopathological examination of the biopsy specimen. Differential diagnostics are required to exclude tumorous processes in the retroperitoneum (sarcomas, haemoblastosis, neuroblastomas, germ cell tumours, metastatic processes from other solid or carcinoid tumours). Similarly, secondary fibrosis from inflammatory processes (sarcoidosis, specific spondylitis or vasculitis, connective tissue disease) must also be excluded. We have diagnosed more than 20 patients with the disease during the last four years of cooperation. From the practical point of view, it is important to stress that the disease responds very well to systemic corticosteroid therapy (Van Bommel et al., 2007). Immunosuppressive therapy is the treatment of choice, and has no alternative. Urological or vascular surgical treatment of the affected renal hollow system or the abdominal aorta, respectively, should be provided as a matter of course. However, systemic corticosteroid monotherapy more often leads to exacerbation of the disease and, therefore, combined immunosuppressive treatment should be preferred, as appropriate.

\section{Conclusion}

IgG4-related diseases are a relatively rare group of conditions, at times with poor clinical symptomatology and a vast variety of involved organs or systems. Its diagnosis and treatment require interdisciplinary cooperation, which has the potential to be the most beneficial for the patient. 


\section{References}

Brito-Zerón, P., Ramos-Casals, M., Bosch, X., Stone, J. H. (2014) The clinical spectrum of IgG4-related disease. Autoimmun. Rev. 13, 1203-1210.

Ebbo, M., Grados, A., Bernit, E., Vely, F., Boucraut, J., Harle, J. R., Daniel, L., Schleinitz, N. (2012) Pathologies associated with serum IgG4 elevation. Int.J. Rheumatol. 2012, 602809.

Kamisawa, T., Zen, Y., Pillai, S., Stone, J. H. (2015) IgG4-related disease. Lancet 385, 1460-1471.

Mahajan, V. S., Mattoo, H., Deshpande, V., Pillai, S. S., Stone, J. H. (2014) IgG4-related disease. Annu. Rev. Pathol. 9, 315-347.

Průcha, M., Kolombo, I., Štádler, P. (2016) Combination of steroids and azathioprine in the treatment of Ormond's disease - A single centre retrospective analysis. Prague Med. Rep. 117(1), 34-41.

Stone, J. H., Brito-Zerón, P., Bosch, X., Ramos-Casals, M. (2015) Diagnostic approach to the complexity of IgG4-related disease. Mayo Clin. Proc. 90, 927-939.

Van Bommel, E. F. H., Siemes, C., Hak, L. E., van der Veer, S. J., Hendriksz, T.T. (2007) Long-term renal and patient outcome in idiopathic retroperitoneal fibrosis treated with prednisone. Am. J. Kidney Dis. 49, 615-625. 